bioRxiv preprint doi: https://doi.org/10.1101/2021.04.29.440827; this version posted April 30, 2021. The copyright holder for this preprint (which

was not certified by peer review) is the author/funder, who has granted bioRxiv a license to display the preprint in perpetuity. It is made available under aCC-BY-NC-ND 4.0 International license.

\title{
Single-cell resolution of MET and EMT programs during zebrafish fin regeneration
}

W. Joyce Tang ${ }^{1,2,3}$, Claire J. Watson ${ }^{1,2}$, Theresa Olmstead ${ }^{1,2}$, Christopher H. Allan ${ }^{1,2}$, Ronald Y. Kwon ${ }^{1,2}$

${ }^{1}$ Department of Orthopaedics and Sports Medicine, University of Washington School of Medicine, Seattle, WA, 98105, USA

${ }^{2}$ Institute for Stem Cell and Regenerative Medicine, University of Washington, Seattle, WA, 98109, USA

${ }^{3}$ Correspondence: wjtang@uw.edu

Further information and requests for resources and reagents should be directed to and will be fulfilled by the Lead Contact, W. Joyce Tang (wjtang@uw.edu). 
bioRxiv preprint doi: https://doi.org/10.1101/2021.04.29.440827; this version posted April 30, 2021. The copyright holder for this preprint (which was not certified by peer review) is the author/funder, who has granted bioRxiv a license to display the preprint in perpetuity. It is made available under aCC-BY-NC-ND 4.0 International license.

\section{SUMMARY}

While humans have limited potential for limb regeneration, some vertebrates can regenerate bony appendages following amputation. During zebrafish fin regeneration, mature osteoblasts at the amputation stump dedifferentiate and migrate to the blastema, where they re-enter the cell cycle and then re-differentiate to form new bone. Osteoblastic cells exhibit dual mesenchymal and epithelial characteristics during fin regeneration, however little is known about why or how this occurs. Using singlecell RNA-sequencing, we found osteoprogenitors are enriched with components associated with the epithelial-to-mesenchymal transition (EMT) and its reverse, mesenchymal-to-epithelial transition (MET). In trajectory analyses, osteoblastic cells solely expressed EMT components, or transiently expressed MET components prior to expressing those for EMT. We found that cdh11, a cancer EMT marker, is expressed during osteoblast dedifferentiation. We also found that esrp1, a regulator of alternative splicing in epithelial cells whose expression is important for MET, is expressed in a subset of osteoprogenitors during outgrowth. This study provides a valuable single cell resource for the study of osteoblast differentiation during zebrafish fin regeneration, and identifies MET- and EMT-associated components which may be important for successful appendage regeneration. 


\section{INTRODUCTION}

While humans have limited potential for limb regeneration, some vertebrates can regenerate bony appendages following amputation. Zebrafish can regenerate amputated caudal fins through the formation of a pool of lineage-restricted progenitors called the blastema [1]. Following fin amputation, mature osteoblasts at the amputation stump dedifferentiate, migrate to the blastema, self-renew, then redifferentiate to form new bone [2-5]. In addition to dedifferentiation of mature cells, osteoprogenitors also derive from resident mesenchymal precursors within the fin ray joint [6]. The formation of osteoprogenitors through dedifferentiation in zebrafish differs from mammals, where osteoblasts primarily derive from resident precursors. The cell biological processes that enable dedifferentiation and reversible switching between differentiated and self-renewing cell states are poorly understood.

Cell plasticity and reversibility between metastable cell states are hallmarks of the epithelial-tomesenchymal transition (EMT), and its reverse, mesenchymal-to-epithelial transition (MET). EMT is a developmental program by which cells lose epithelial (E) characteristics (e.g., E-cadherin, desmosomes, cortical actin, apical-basal polarity) while adopting features of mesenchymal (M) cells (e.g., increased vimentin) [7]. EMT is often observed during cancer invasion and metastasis, where it results in dedifferentiation and acquisition of cancer stem cell-like properties [8]. While cancer progression is impaired by complete EMT, cancer cell stemness is enhanced by partial EMT, where cells reversibly switch between metastable cell states with hybrid E/M or M/E phenotypes [9]. Epimorphic regeneration exhibits a number of biological parallels to the progression of solid tumors. It has been suggested that certain mechanisms enabling regeneration may be co-opted by cancer to promote growth at primary and metastatic sites [10]. However, previous single cell RNA-seq analyses have failed to identify EMT signatures during axolotl limb regeneration [10-12].

In mammals, early osteoblastic cells are stromal cell-like in appearance, whereas matrix-secreting osteoblasts are tall or cuboidal and form a continuous layer, bearing a partial resemblance to epithelial cells [13]. The primary form of cell-cell adhesion between osteoblasts is through adherens junctions [14, 15], which are typically found in epithelial and endothelial cells and rely on the interaction of cadherins at the cell surface to form an intercellular adhesion complex. Multiple studies have shown that interfering with cadherin function impairs osteoblast differentiation [16]. Upon further maturation, active osteoblasts transition into quiescent bone lining cells, appearing as flat and elongated cells on the mineralized surface. Thus, while mesenchymal in origin, osteoblastic cells differentially exhibit characteristics of mesenchymal and epithelial cells depending on cell state.

During zebrafish fin regeneration, Stewart et al. noted dual mesenchymal and epithelial characteristics in osteoblastic cells, and hypothesized this was due to EMT [17]. Specifically, they observed that blastemal osteoprogenitors expressed the EMT-associated transcription factors (EMT-TFs) twist2 and twist3, loss of the epithelial marker $\alpha$-catenin 1, and changed from an elongated to a polygonal morphology. However, this process remains poorly understood. Only a limited set of EMT markers have been characterized, which may be insufficient to resolve hybrid cells that exhibit both $\mathrm{E}$ and $\mathrm{M}$ characteristics [7]. Moreover, multiple markers are often needed to precisely assess the state of a cell undergoing EMT within specific biological contexts [7]. Finally, many transcription factors and other components critical for EMT also affect other cellular functions. These components may be regulated through programs that involve canonical EMT [7]. As such, more in-depth molecular characterizations are needed. While single cell analyses are comprehensive approaches and can provide valuable insight, previous single-cell RNA-seq (scRNA-seq) studies of zebrafish caudal fin regeneration have reported low numbers of osteoblastic cells [18], preventing an in-depth analysis of $\mathrm{E}$ and $\mathrm{M}$ characteristics.

In this study, we performed sci-RNA-seq (single-cell combinatorial indexing RNA sequencing) [19] during zebrafish caudal fin regeneration. We found that distinct osteoprogenitor populations are enriched with components associated with the epithelial-to-mesenchymal transition (EMT) and its reverse, mesenchymal-to-epithelial transition (MET). Pseudotemporal analysis indicated two trajectories: one in which osteoblastic cells solely express EMT components, and a second in which they transiently express MET components prior to expressing those for EMT. We found that $c d h 11$, a cancer EMT marker [20], is expressed during osteoblast dedifferentiation. We also found that esrp1, an epithelial cell-enriched 
regulator of alternative splicing whose expression is important for MET [21], is expressed in a subset of osteoprogenitors during outgrowth. Thus, zebrafish fin regeneration employs MET- and EMT-associated components which may be important for successful appendage regeneration.

\section{RESULTS \\ sci-RNA-seq captures rare osteoblastic populations}

We performed sci-RNA-seq using 225 fins from 3-5 days post amputation (dpa), which yielded 19,603 nuclei after filtering for quality control. At these timepoints, a variety of tissues have regenerated (Fig 1A). Cells showed similar characteristics across timepoints (Figure 1 - Supplemental Figure 1) and thus were pooled for final analysis. Unbiased clustering identified 12 different cell populations, of which we defined 10 out of 12 by cell/tissue type using published biomarkers (Fig 1BC, Figure 1 Supplemental Table 1). We identified two populations of osteoblastic cellsosteoblast 1 ( 973 cells) and osteoblast2 (700 cells)—marked by the strong expression of the osteoprogenitor marker runx2a/b (Fig 1C), a transcription factor expressed during early osteoblast differentiation. Mapping of osteoblast1 and osteoblast2 identities to a previous scRNA-seq dataset of fin regeneration [18] revealed 16 and 23 osteoblast 1 and osteoblast2 cells, respectively, indicating the difficulty of capturing osteoblastic cells in scRNA-seq studies.

Osteoprogenitors can differentiate into osteoblasts and joint-forming cells [22] (Fig 1D). Cells within the osteoblast1 cluster expressed genes known to be upregulated during osteoprogenitor differentiation into joint cells. This included pthlha, a marker of joint cells [7], and $m m p 9$, a marker of joint-derived resident osteoprogenitors [6] (Fig 1E). In contrast, the osteoblast2 cluster expressed genes known to be specifically expressed during osteoblast differentiation, notably col10a1a/b (a marker of pre-osteoblasts), sp7 (a marker of immature osteoblasts), and ihha [7] (Fig 1E). The osteoblast1 and osteoblast2 clusters scored higher for joint cell and osteoblast gene signatures, respectively (Fig 1E). We identified genes with significant differences in expression between the two osteoblast groups (Fig 1 - Supplemental Table 2). These genes included $s / c 8 a 4 b$ in the osteoblast2 cluster (Fig 1F), and Imx1ba in the osteoblast1 cluster (Fig 1G). We also identified genes

A

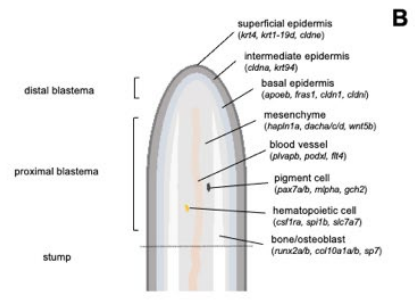

C

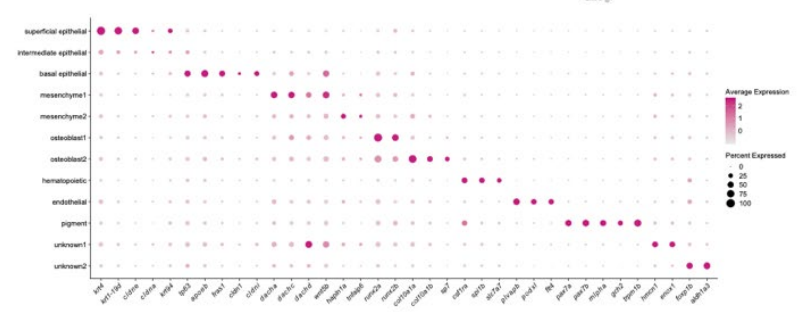

D

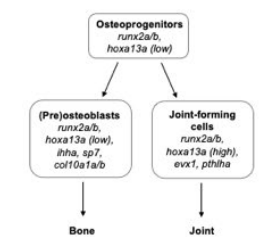

E
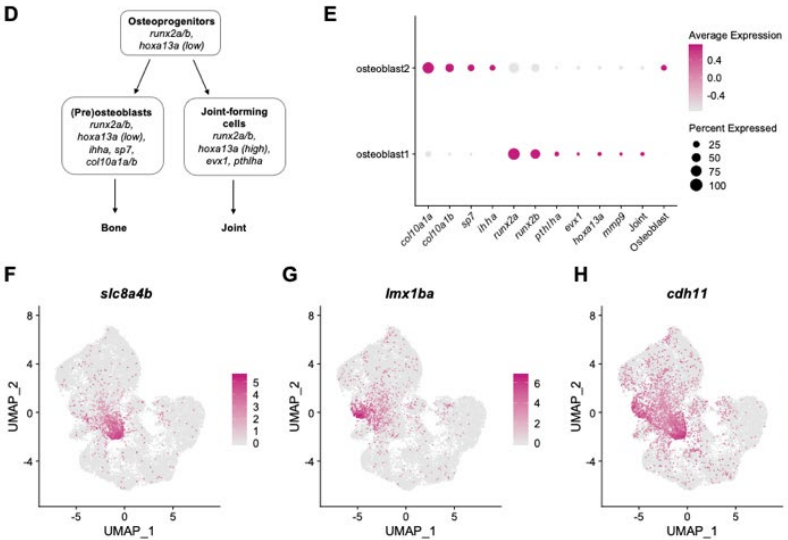

G
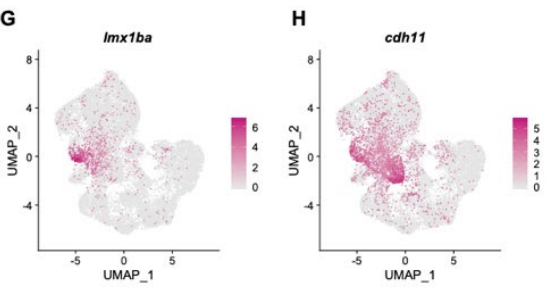

Fig 1. Molecular profiling of fin regeneration reveals two osteoblast populations. (A) Schematic of zebrafish fin regeneration showing tissues and corresponding gene markers. (B) UMAP plot visualizing 19,603 cells from sci-RNA-seq analysis of regenerated fins at 3 and $5 \mathrm{dpa}$. Of the 12 distinct clusters, 10 tissue types could be identified by molecular markers, including two osteoblast populations. (C) Dotplot visualizing expression of genes demarcating the 12 cell clusters. Circle size represents the percentage of cells expressing the gene, and color indicates average gene expression. (D) Schematic of osteoprogenitor differentiation into either osteoblasts or joint-forming cells and corresponding gene markers (adapted from McMillan et al., Development 2018). (E) Dotplot comparing the expression of joint cell markers and bone markers in the two osteoblast populations. The clusters osteoblast 1 and osteoblast2 are enriched with genes associated with joint-forming cells and osteoblasts, respectively. (FH) Feature plots for s/c8a4b, Imx1ba, and cdh11. 
both groups, compared to non-osteoblastic cell types (Fig 1 - Supplemental Table 3). This included cdh11 (Fig $1 \mathrm{H})$, which was recently identified by scRNA-seq to be a potential marker of osteoblastic cells, but still remains to be validated [18]. We concluded that osteoblast1 and osteoblast2 were enriched for cells fated to become osteoblasts and joint cells, respectively.

\section{Osteoblastic cells exhibit distinct cell states and trajectories}

We subset the osteoblast2 cells for further analysis. Unbiased clustering of the osteoblast2 cells revealed five subclusters (Fig 2A). Analysis of markers of osteoblast differentiation (Fig 2B) and cell cycle phase (Fig $2 \mathrm{C}$ ) revealed distinct cell cycle and differentiation states. Clusters 4 and 5 had the highest osteoblast differentiation scores. Whereas cluster 5 was enriched for $s p 7$, cluster 4 was enriched for col10a1a/b. Moreover, cluster 5 had the lowest percentage of cells in the G2/M and S phases. Cluster 3 had a lower osteoblast differentiation score compared to clusters 4 and 5 . Moreover, these cells had relatively high expression of col10a1a/b, and had the highest percentage of cycling cells as indicated by cells in the $\mathrm{G} 2 / \mathrm{M}$ and $S$ phases. Cluster 3 also had low runx2a/b expression, which is in agreement with previous studies showing that murine RUNX2 levels regulate cell cycle entry and exit in osteoblasts, with minimal levels of RUNX2 during $\mathrm{G} 2$ and mitosis $[23,24]$. Clusters 1 and 2 had the lowest osteoblast differentiation scores, and had higher runx2a/b expression compared to clusters 3 and 4 . The above studies identify diverse osteoblastic subpopulations which differ in cell cycle and differentiation status, three of which (clusters 3, 4, and 5) we could identify based on cell cycle status or known osteoblastic biomarkers.
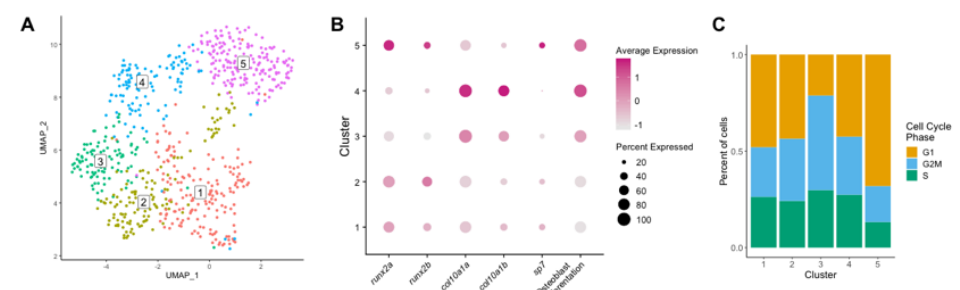

D

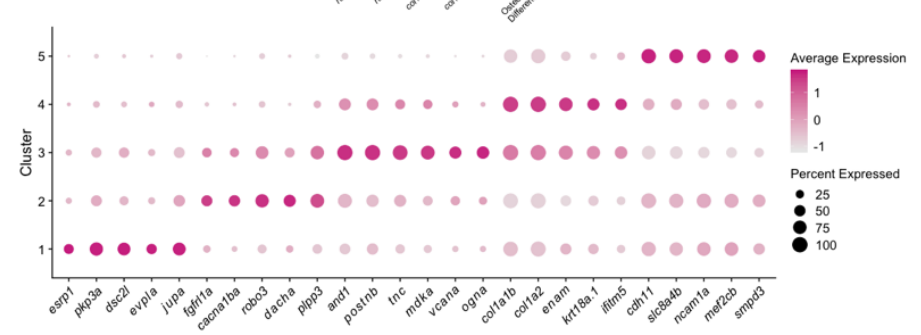
Gene

E
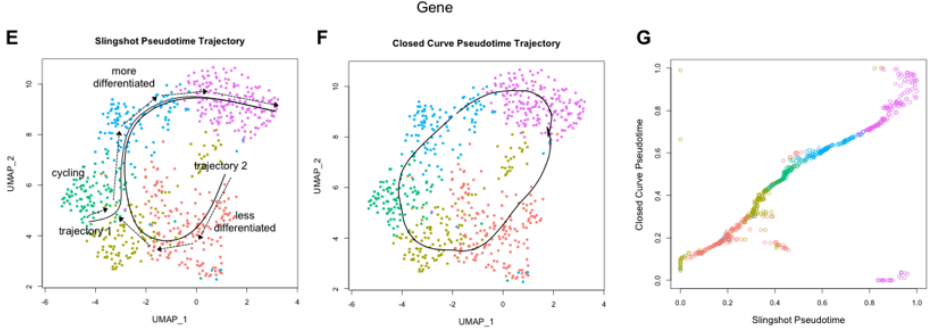

Fig 2. Clustering and trajectory analysis of osteoblast2 population. (A) UMAP plot showing five distinct osteoblastic subclusters. (B) Dotplot visualizing expression of known genes marking the different stages of osteoblast differentiation and osteoblast differentiation scores. (C) Percentage of cells in each cell cycle phase for each osteoblast subcluster. (D) Dotplot showing selected genes found to be differentially expressed within each osteoblastic subcluster. (E-G) Pseudotemporal ordering of osteoblast2 cells. (E) Slingshot pseudotime ordering yields two trajectories. Trajectory 1 starts in cluster 3 , whereas trajectory 2 starts in cluster 1. (F) Closed curve analysis suggests a similar trajectory to trajectory 2 derived from Slingshot analysis. (G) Comparison of the closed curve trajectory to Trajectory 2 derived from Slingshot shows that the orderings from open (Slingshot) and closed curve analyses are highly similar.

Next, we performed differential gene expression analysis (Fig 2D, Supplemental Table 1) and found that osteoblast2 subclusters were uniquely characterized by genes associated with the epithelium or involved in extracellular matrix formation or osteoblast differentiation. Of the 10 most differentially expressed genes in cluster 1, nine were associated with epithelial cells, including genes encoding for components of desmosomes ( $d s c 2 l$ and evpla) and adherens junctions ( $p k p 3 a)$. Among the top marker genes in clusters 3-4 were those encoding for extracellular matrix components. For instance, 10 of the top 20 genes of cluster 3 included markers such as postnb, and1, tnc, vcana, and ogna, while the top two most differentially expressed genes in cluster 4 encoded for Type I collagen (col1a1b and col1a2). Cluster 4 was also marked by the expression of ifitm 5 which has been implicated in skeletal disease [25]. The top five markers of cluster 5 included genes whose orthologs are expressed during osteoblast differentiation (cdh11 [14], mef2c [26], ncam1a [27]) and/or are implicated in skeletal disease (smpd3 [28]). A complete analysis of enrichment for pathways and GO terms can be seen in Fig 2 - Supplemental Figure 1. 
To assess ordering through cell states, we performed pseudotime analysis. Unbiased analysis using Slingshot [29] revealed two trajectories (Fig 2E). Trajectory 1 started in cluster 3 and progressed through clusters 4 and 5 . In this trajectory, cycling osteoprogenitors transition into pre-osteoblasts that express col10a1a/b, and then further transition into immature osteoblasts that express sp7. Trajectory 2 started in cluster 1 , and then progressed through a trajectory similar to trajectory 1 . In both trajectories, extracellular matrix genes such as postnb, and1, tnc, vcanb, and ogna are expressed prior to or concomitantly with genes encoding for Type I collagen (col1a1b and col1a2). This is consistent with the synthesis of a transitional matrix rich in tenascin $\mathrm{C}$ prior to the synthesis of ECM proteins characteristic of differentiated skeletal tissues like collagen type I [30] during zebrafish fin regeneration and salamander limb regeneration. These studies point to multiple candidate trajectories underlying the identified cell states.

We then evaluated whether the trajectories exhibited characteristics of de- and redifferentiation. De- and redifferentiation involves a cyclical progression through cell states which would manifest as a closed curve through gene expression space. We therefore sought to examine whether trajectory 2 represented a path through cellular de- and redifferentiation by assessing if the pseudotime orderings in the trajectory were similar to those derived from a closed curve. Algorithms for the analysis of cyclic trajectories are sparse compared to non-cyclic methods [31]. Moreover, ANGLE, the highest performing cyclic algorithm benchmarked in [31], requires 2D data embeddings. We therefore devised an algorithm for pseudotime inference that enables closed curve trajectory analysis using data embeddings of arbitrary dimension (Fig $2 \mathrm{~F}$ ). Pseudotime orderings derived from the closed curve trajectory were highly similar to trajectory 2 (Fig $2 \mathrm{G}$ ). In addition, orderings derived using either UMAP or PCA embeddings were comparable to each other (Fig 2 - Supplemental Figure 2). Thus, pseudotime orderings were similar whether using an open (Slingshot) or closed curve, the latter of which is predicted with a cyclical progression through cellular deand redifferentiation states.

\section{Osteoblastic cells differentially express MET- and EMT-associated genes}

The top markers for osteoblastic cell clusters included canonical markers for MET and EMT. For instance, the second most differentially expressed gene in cluster 1 was esrp1. Epithelial splicing regulatory proteins (ESRP) are highly conserved RNA-binding proteins that regulate alternative splicing, and which participate in MET and EMT [32, 33]. There are two paralogs of esrp in vertebrates, ESRP1 and ESRP2. They are required for an epithelial splicing program involving alternative splicing of hundreds of genes, many of which have functions in cell-cell adhesion, cell polarity, and cell migration [34]. This includes Fgfr2; Esrp1 and Esrp2 are required for the epithelial isoform of Fgfr2, and knockdown of Esrp results in the complete switch to the mesenchymal isoform of Fgfr2 [32]. In zebrafish, double knockout of esrp1 and esrp2 leads to multiple developmental abnormalities, including malformed fins [35].

The most differentially expressed gene in cluster 5 was $c d h 11$. CDH11 is expressed during cadherin switching in EMT [36]. In this process, expression of $\mathrm{CDH} 1$ (epithelial cadherin) decreases in concert with increased expression of $\mathrm{CDH} 11$ and $\mathrm{CDH} 2$ (mesenchymal cadherins). As such, $\mathrm{CDH} 11$ serves as a biomarker of EMT [37]. In regard to its embryonic functions, $\mathrm{CDH} 11$ is predominantly expressed within tissues of mesodermal origin [38], and is linked to morphogenesis in the head, somite, and limb bud of early mouse embryos [39]. In addition to its expression within mesenchymal cells, Cdh11 is also expressed in parts of the neural tube and otic vesicle in early mouse embryos [39]. In zebrafish, knockdown of Cdh11 results in otic vesicle [40] and retinal defects [41]. In chick, CDH11 expression is observed during neural crest cell delamination, which exhibits characteristics of EMT [42]. This motivated us to further explore the mesenchymal and epithelial characteristics of osteoblastic cells.

First, we computed mesenchymal and epithelial differentiation scores (Fig 3A). Whereas mesenchymal differentiation scores were relatively uniform across cell clusters, epithelial differentiation scores were higher in clusters 1-3 compared to clusters 4-5. As a consequence, epithelial scores were higher than mesenchymal scores in clusters 1-3, while mesenchymal scores were higher than epithelial scores in clusters 4-5. Hence, osteoblastic cells express genes associated with both mesenchymal and epithelial differentiation, which are differentially expressed depending on cell state. 
We tested the hypothesis that EMT- and MET-like transitions underlie transitions between mesenchymal- and epithelial-like cell states in osteoblastic cells. Partial MET and EMT result in intermediate E/M and $\mathrm{M} / \mathrm{E}$ states which exhibit dual epithelial and mesenchymal characteristics (Fig 3B), necessitating the analysis of multiple markers. We first assessed canonical markers and transcription factors associated with MET and EMT (Fig 3C-G). Cluster 1 had the highest expression of the epithelial markers esrp1 and $c d h 1$, whereas clusters 3 or 5 had the highest expression of the mesenchymal markers $c d h 2 / 11$, prrx $1 b$, twist $2 / 3$, ctnna $2 / b 1 / b 2$, and $z e b 1 b / 2 a$. The expression of twist $2 / 3$ and prrx $1 b$ was highest in cluster 3 . These cells match the description of twist+ osteoprogenitors in the blastema identified by Stewart et al. [17], and which were hypothesized to be undergoing EMT. The expression of zeb1b/zeb2a was highest in cluster 5 . Consistent with the notion that ZEB drives a mesenchymal identity partly by repressing ESRP [43], we found strongest expression of the zeb1b/zeb2a paralogs in cluster 5 , where there is weakest esrp1 expression. These data indicate that canonical MET and EMT components are expressed in osteoblastic cells.

To assess whether Esrp was active during fin regeneration, we examined alternative splicing of known ESRP-dependent genes using VAST-TOOLS [44]0. Because our method of scRNA-seq is biased toward the 3 ' end of transcripts, which may miss splicing activity that occurs near the 5 ' end, we analyzed bulk RNAseq datasets of fin regeneration from Lee et al. in which paired-end libraries were sequenced [45]. Analysis detected ESRP-dependent alternative splicing in fgfr2, namely an increase in the epithelial isoform and decrease in the mesenchymal isoform, at $1 \mathrm{dpa}$ (Fig 3 -Supplemental Figure 1). Within this same dataset, RNAseq was performed on $s p 7+$ cells [45]. In this dataset, we identified 1144 alternative splicing events (Fig 3 - Supplementary Table 1). While we did not detect the alternative splicing of fgfr2, of the 1144 alternative splicing events that were found, 110 were identified to be dependent on esrp1/2 in zebrafish (Fig 3 - Supplementary Table 2) [35]. We concluded that esrp1 splicing activity occurs during fin regeneration.

We next assessed whether osteoblastic cells express MET and EMT gene signatures. We constructed an orthologous EMT gene signature based on a hallmark gene set from the Molecular Signatures Database [46]. For MET, we constructed an orthologous signature based on a gene set derived from human prostate and breast cancer cell lines induced to undergo MET via OVOL1/2 expression [47]. Scoring revealed that cluster 3 had the strongest scoring for the EMT gene signature, whereas cluster 1 had the strongest scoring for the MET gene signature (Fig 3C). We also identified enrichment of these signatures in mesenchymal cell subclusters (Fig 3 -Supplemental Figure 2). Differential gene expression analysis 
bioRxiv preprint doi: https://doi.org/10.1101/2021.04.29.440827; this version posted April 30, 2021. The copyright holder for this preprint (which was not certified by peer review) is the author/funder, who has granted bioRxiv a license to display the preprint in perpetuity. It is made available under aCC-BY-NC-ND 4.0 International license.

revealed enrichment of MET and EMT genes in distinct osteoblast subclusters (Fig 3H). The top EMT markers in cluster 3 were enriched in genes associated with extracellular matrix, including matrix components (tnc, col6a2, col5a1, col1a2, col16a1, col6a3, fbln5, fbln2), collagen-binding products (tgbi, lum, dcn), and matrix remodeling ( $\mathrm{mmp} 2$ ). Thus, MET- and EMT-associated gene signatures are expressed in osteoblastic cells during fin regeneration. Finally, to elucidate cell state transitions, we ordered the osteoblastic cells in pseudotime and scored for MET and EMT gene signatures (Fig $3 \mathrm{I}$ ). In trajectory 2, osteoblastic cells express MET-associated genes prior to expressing EMT-associated genes.

Taken together, our studies indicate that osteoblastic cells express canonical MET and EMT components, as well as MET- and EMT-associated gene signatures. Moreover, we have identified multiple candidate trajectories underlying transitions between cell states.

\section{Osteoprogenitors express cdh11 and esrp1 in situ}

To place our findings into a spatiotemporal context, we performed dual RNA in situ hybridization (ISH) for cdh11 and esrp1. In general, staining for cdh11 was highest in cells within the mesenchymal compartment, whereas staining for esrp1 was highest in epithelial cells, with decreasing expression in the deeper epidermis layers (intermediate and basal epidermis) (Fig 4A, 4B, 4C).

We examined several regenerative stages. We first examined fins from $1 \mathrm{dpa}$ (Fig 4A), a timepoint at which a wound epithelium is present but a blastema is not visible. Prior studies have shown that at this timepoint, osteoblastic dedifferentiation and migration has initiated [2]. Staining for cdh11 was observed in bone lining cells near the amputation stump (Fig 4A1, black arrow), with decreased staining in bone lining cells in more proximal locations (Fig 4A1 orange arrows). We also observed staining for cdh11 in cells near the fin ray joints (Fig 4A1, red arrowhead), a location known to harbor joint-derived resident osteoprogenitors [6]. Finally, staining for cdh11 was observed in cells aligned with the amputation plane, some of which exhibited a rounded morphology (Fig 4A2, stars). Staining for esrp1 in the mesenchymal compartment was not observed. Thus, at $1 \mathrm{dpa}$, staining for cdh11 was present in cells
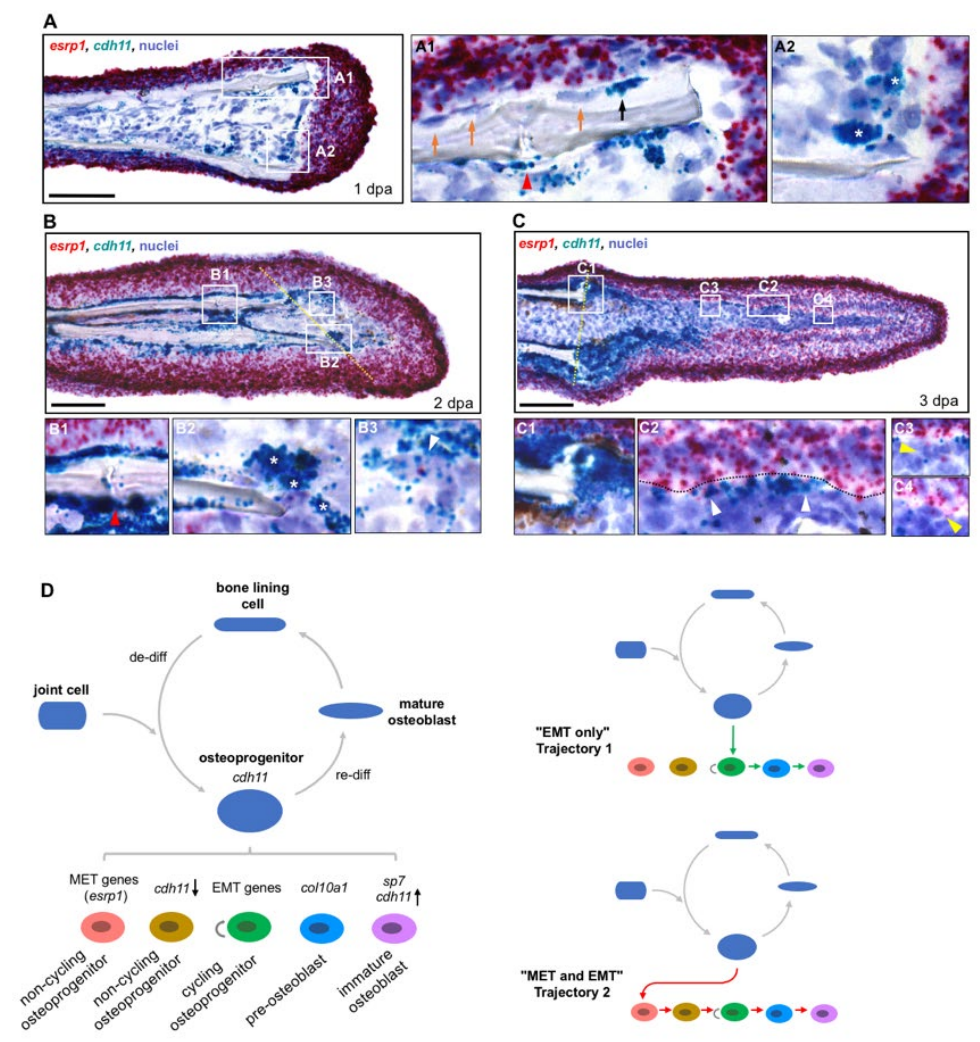

Fig 4. Osteoprogenitors express esrp1 and cdh11 in a temporal pattern during fin regeneration. (A-C) Dual RNA ISH hybridization on cryosections from 1, 2, and 3 dpa fin regenerates using RNAScope. All scale bars are $60 \mu \mathrm{m}$. Amputation plane is indicated with dashed yellow line. (A) Expression of esrp1 and cdh11 in a $1 \mathrm{dpa}$ fin cryosection. (A1 inset) cdh11 only stained some cells lining the bone (black arrow, positive staining; orange arrows, no staining). cdh11 staining was also observed in cells around the fin ray joints (red arrowhead). (A2 inset) Cells staining for cdh11 were observed along the amputation plane, lining the distal border of the mesenchymal compartment (stars). (B) Expression of esrp1 and cdh11 in a 2 dpa fin cryosection. (B1 inset) cdh11-positive cells were prominent along the fin bone, including near the fin joint (red arrowhead). (B2 inset) Multiple cdh11-positive cells were observed near the amputation stump (stars). (B3 inset) Arrowhead indicates a cell staining positive for both esrp1 and cdh11 in the forming blastema. (C) Expression of esrp1 and cdh11 in a 3 dpa fin cryosection. (C1 inset) Prominent cdh11 expression is observed at the amputation stump. (C2 inset) Multiple cells showing staining for both cdh11 and esrp1 (arrowheads). (C3, C4 insets) Multiple cells in the lateral mesenchymal compartment were positive for esrp1 but not $c d h 11$ (yellow arrowheads). (D) Model of osteoblastic de- and redifferentiation during fin regeneration (left). The two trajectories as predicted by Slingshot are shown at right. 
located at sites that harbor dedifferentiating osteoblasts and resident osteoprogenitors, whereas staining for esrp1 was absent from these cells.

Next, we examined fins from $2 \mathrm{dpa}$, a timepoint in which a forming blastema is visible. Staining for cdh11 was noticeably increased from $1 \mathrm{dpa}$ (Fig 4B). We observed increased staining in cells near the amputation stump (Fig 4B2, stars) as well as in cells near the fin ray joints (Fig 4B1, red arrowhead). Within the blastema, staining for cdh11 was present in the lateral mesenchymal compartment (Fig 4B3). We observed staining for esrp1 in several cells within the lateral mesenchymal compartment, and which co-stained with cdh11 (Fig 4B3, white arrowhead). Thus, at 2 dpa, staining for cdh11 was present in cells within the blastema that were likely to be osteoprogenitors, with staining for esrp1 present in some of these cells.

Finally, we examined fins from $3 \mathrm{dpa}$. At this timepoint, newly regenerated bone is visible, with more mature osteoblastic cells residing near the amputation stump, and less mature osteoprogenitors located distally [48]. Staining for cdh11 was increased from 2 dpa (Fig 4C). We observed prominent staining in cells lining the amputation stump (Fig 4C1), and in the lateral mesenchymal compartment where new bone was likely to form (Fig 4C2). Staining for cdh11 generally decreased in the distal direction. In regard to esrp1, we observed increased staining in cells within the mesenchymal compartment compared to 2 dpa. Staining for esrp1 was observed in cells in the lateral mesenchymal compartment in both proximal (Fig 4C3) and distal (Fig 4C4) locations. Within these cells, some co-staining of esrp1 and cdh11 was observed (Fig 4C2, white arrowheads). Notably, we did not observe a clear compartment of esrp1+ mesenchymal cells. Rather, esrp1+ cells were relatively sparse, and intercalated among or near cdh11+ cells in both proximal and distal locations within the blastema. Similar findings were observed when performing dual RNA ISH for esrp1 and runx2 (Fig 4 - Supplemental Figure 1). Thus, similar to 2 dpa, at $3 \mathrm{dpa}$, staining for $c d h 11$ was present in cells likely to be osteoprogenitors, with some of these cells staining for esrp1.

We used our data to develop a model of osteoblastic de- and redifferentiation, which we hypothesize involves two trajectories (Fig 4D). The first trajectory, called the "EMT only" trajectory, is based on trajectory 1 and excludes cluster 1 cells that express MET-associated genes. In this trajectory, following fin amputation, dedifferentiating bone lining cells and resident progenitors express cdh11. These cdh11+ osteoprogenitors delaminate and migrate into the blastema, a behavior similar to epithelial cells undergoing EMT. There, they enter into the cell cycle, express EMT-associated gene signatures, and differentiate into osteoblasts. Thus, in the "EMT only" trajectory, osteoblasts acquire a mesenchymal-like cell state through a process that employs programs similar to EMT, as proposed by Stewart et al [17].

The second trajectory, called the "MET and EMT" trajectory, is based on trajectory 2 and includes cluster 1 cells that express MET-associated genes. This trajectory begins similarly as the "EMT only" trajectory. Specifically, dedifferentiating bone lining cells and resident progenitors become $c d h 11+$ osteoprogenitors that delaminate and migrate to the blastema. However, rather than completing EMT, a subset of cdh11+ osteoprogenitors express esrp1 and other MET-associated components, transition into the cluster 2 cell state, and then express EMT-associated gene signatures. These osteoprogenitors then differentiate into osteoblasts. Because esrp1+/cdh11+ osteoprogenitors were relatively sparse (comprising $14.2 \%$ of all osteoblast2 cells in our sci-RNA-seq analyses), we hypothesize that the "EMT only" trajectory is predominant, whereas the "MET and EMT" trajectory serves as a secondary path to generate specialized cells within the blastema.

\section{DISCUSSION}

In summary, we found that MET- and EMT-associated components are enriched in osteoprogenitor populations. This includes $c d h 11$, a cancer EMT marker, which is expressed during osteoblast dedifferentiation. This also includes esrp1, whose expression is important for MET [21], in a subset of osteoprogenitors during outgrowth. Thus, zebrafish fin regeneration employs MET- and EMT-associated components which may be important for successful appendage regeneration. 
Some limitations of our study should be considered. First, gene expression alone is insufficient to indicate that MET or EMT is occurring [7]. In our studies, osteoblastic cells expressed several EMT-TFs (including orthologs for ZEB and TWIST, part of a core group of transcription factors that are expressed in all instances of EMT [7]), and were enriched with EMT-associated genes. However, we were unable to assess cell phenotypes that define EMT such as loss of apical-basal polarity, modulation of the cytoskeleton, and cell-cell adhesive properties. Thus, further studies are needed to determine whether the expression of MET- and EMT-associated components in our study are reflective of actual MET and EMT, or are distinct processes that bear resemblance to MET and EMT. Another limitation of our study is the focus of our sci-RNA-seq analysis on a later window of regeneration (3-5 dpa). While this prevented transcriptomic examination of earlier timepoints, our RNA ISH studies allowed us to connect our single cell dataset to early osteoprogenitor states. Finally, it should be emphasized that the trajectories constructed in this study are solely based on unbiased inference, and that lineage tracing studies are needed to test their predictions.

By identifying EMT and MET components expressed during fin regeneration, our studies provide a useful lens to identify pathways underlying de- and redifferentiation. In our studies, paralogs for zeb and twist (but not snail) were markedly expressed in osteoblastic cells. Moreover, Stewart et al. observed twist2/3 expression near the amputation stump at $1 \mathrm{dpa}$ [17], similar to the spatiotemporal expression we observed for cdh11. Thus, osteoblastic dedifferentiation may involve expression of twist, zeb, and cdh11, a combination that has been observed in certain EMT-like processes [42, 49]. Further studies are also needed to determine whether osteoblastic cells exhibit reversibility between metastable M/E- and E/M states, reminiscent of that associated with increased cancer cell stemness [9].

A growing body of studies suggests that EMT or MET pathways may be harnessed to engineer or enhance dedifferentiation in mammalian cells. For instance, early stages of induced pluripotent stem cell (iPSC) reprogramming from mouse embryonic fibroblasts require MET and transition to an epithelial-like morphology [50]. Esrp1 has been shown to enhance reprogramming of fibroblasts to iPSCs, in part by alternative splicing of the transcription factor Grhl1 (grainyhead like transcription factor 1) [51]. Reprogramming efficiency is also increased by early induction of EMT [52, 53]. Moreover, increased differentiation potential of breast epithelial cells similar to mesenchymal stem cells is achieved by overexpression of Snail or Twist $[53,54]$. Current studies are focused on further elucidating MET pathways and EMT components expressed in osteoblastic cells during zebrafish fin regeneration, and how MET and EMT pathways can be harnessed to engineer cyclic de- and redifferentiation for stem cellbased therapies.

\section{METHODS}

$\underline{\text { Tissue collection }}$

Approval for this project was granted by the University of Washington IACUC. Adult wildtype (AB) zebrafish were anesthetized with MS-222 and subjected to $50 \%$ amputation of the caudal fin and allowed to recover. At various days post amputation (dpa), regenerated fin tissues were collected and either fixed overnight at $4{ }^{\circ} \mathrm{C}$ in $10 \%$ neutral-buffered formalin for cryosectioning, or immediately frozen at $-80^{\circ} \mathrm{C}$ for sequencing. Nuclear extraction, RNA isolation, library construction, and sequencing for sci-RNA-seq were performed as previously described [19].

\section{scRNA-seq analysis}

A pilot dataset from 25 fins collected at 3 dpa was analyzed with Seurat using the standard recommended workflow for quality control filtering $(200<$ nFeatures_RNA < 2500) and data processing [55]. Unbiased clustering was performed with the top 15 principle components (PCs) and with clustering resolution set to 0.5. To assign tissue types to the resulting clusters, the FindAllMarkers function was used for differential gene expression analysis to identify gene markers specifically expressed by each cluster. The tissue type annotations of the pilot dataset were then projected onto the larger dataset of 3 and 5 dpa fins $(n=200)$, using the FindTransferAnchors and TransferData functions. The two datasets were then combined, and the standard quality control and data processing workflow was then repeated (number of PCs $=15$, resolution $=0.5$ ). For subclustering of the osteoblast population, we subset the osteoblast 2 cells from the 
combined dataset and again applied the standard Seurat data processing workflow (number of PCs $=15$, resolution $=0.8$ ). All plots were generated in $\mathrm{R}$ with either Seurat, base $\mathrm{R}$ graphics, or with the dittoSeq package [56].

Trajectory analysis

Trajectory analysis was performed using Slingshot [29] in an unsupervised manner using UMAP embeddings, or with a custom $\mathrm{R}$ script.

Alternative splicing analysis

The Vertebrate Alternative Splicing and Transcription Tools (VAST-TOOLS) software [44] was used to profile alternative splicing events in bulk RNA-seq data (GSE126701) [45]. Data from experimental replicates were merged, and differential splicing analysis was performed using the "danRer10" library of alternative splicing profiles in zebrafish as reference (downloaded from the VastDB site, https://vastdb.crg.eu/wiki/Main_Page). All comparisons were performed with the "compare" module and implemented with the following parameters: --min_dPSI 15 --min_range 5 --p_IR. A custom R script was used to compare the alternative splicing events against those that were previously annotated as esrpdependent [35].

\section{Cryosectioning}

Fixed tissues were washed in 1X PBS/0.05\%Tween20 (PBS-T) for $3 \times 5$ minutes, then dehydrated in increasing graded concentrations of methanol and stored at $-20^{\circ} \mathrm{C}$ for up to 6 months. Tissues were processed for embedding by first rehydrating to PBS-T in increasing graded concentrations, then cryoprotected by incubation in $15 \%$ sucrose/1X PBS for 1 hour at room temperature, followed by $20 \%$ sucrose/1X PBS for 4 hours to overnight at $4^{\circ} \mathrm{C}$. Embedding was done in O.C.T. compound (Fisher Scientific) in Peel-A-Way embedding molds (Polysciences, Inc.) and snap frozen in either dry ice or liquid nitrogen and stored at $-80^{\circ} \mathrm{C}$ until ready for sectioning. $\sim 15 \mu \mathrm{m}$ sections were made on a Leica CM1850 cryostat, collected onto charged slides and allowed to dry for several hours at room temperature before being stored at $-20^{\circ} \mathrm{C}$.

In situ hybridization with RNAscope

Custom and catalog probes were purchased for esrp1, runx2a, and cdh11 from the manufacturer (ACD Bio). In situ hybridization was performed using the RNAScope $2.5 \mathrm{HD}$ Duplex Detection kit essentially as per manufacturer instructions, with the following modifications: sections were treated with Target Retrieval solution in a steamer for 8 minutes at $\sim 100^{\circ} \mathrm{C}$, and permeabilized with Protease Plus for 15 minutes at $40^{\circ} \mathrm{C}$.

Imaging and analysis

Slides were imaged with a 40X objective on an Aperio VERSA 200 slide scanner. All images are representative of at least two different sections. Sections for 1 and 2 dpa analysis were from a single ISH experiment, and sections for $3 \mathrm{dpa}$ analysis were from three separate experiments. Per the manufacturer's recommendation, more than one spot per ten cells in a field of view at 40X magnification was considered positive staining. We verified this criterion using a negative control.

Data availability

All raw and processed sci-RNA-seq data will be submitted to the Gene Expression Omnibus repository upon acceptance.

\section{ACKNOWLEDGEMENTS}

The authors would like to acknowledge Dr. Ting Wang for generous sharing of scRNA-seq data, as well as members of the MSBL, the UW Regeneration Club, and Dr. Lauren Saunders for helpful discussions. Research reported in this publication was supported by the Brotman Baty Institute Seattle Single Cell Initiative, a Seed Grant from the University of Washington Department of Orthopaedics and Sports Medicine, and the National Institute of Arthritis and Musculoskeletal and Skin Diseases of the National 
bioRxiv preprint doi: https://doi.org/10.1101/2021.04.29.440827; this version posted April 30, 2021. The copyright holder for this preprint (which was not certified by peer review) is the author/funder, who has granted bioRxiv a license to display the preprint in perpetuity. It is made available under aCC-BY-NC-ND 4.0 International license.

Institutes of Health under Award Number AR066061. The content is solely the responsibility of the authors and does not necessarily represent the official views of the National Institutes of Health. 


\section{REFERENCES}

1. Poss, K.D., J. Shen, A. Nechiporuk, G. McMahon, B. Thisse, C. Thisse, and M.T. Keating, Roles for Fgf signaling during zebrafish fin regeneration. Dev Biol, 2000. 222(2): p. 347-58

2. Knopf, F., C. Hammond, A. Chekuru, T. Kurth, S. Hans, C.W. Weber, G. Mahatma, S. Fisher, M. Brand, S. Schulte-Merker, and G. Weidinger, Bone regenerates via dedifferentiation of osteoblasts in the zebrafish fin. Dev Cell, 2011. 20(5): p. 713-24

3. Singh, S.P., J.E. Holdway, and K.D. Poss, Regeneration of amputated zebrafish fin rays from de novo osteoblasts. Dev Cell, 2012. 22(4): p. 879-86. PMC3341140

4. Stewart, S. and K. Stankunas, Limited dedifferentiation provides replacement tissue during zebrafish fin regeneration. Dev Biol, 2012. 365(2): p. 339-49. PMC3370987

5. Sousa, S., N. Afonso, A. Bensimon-Brito, M. Fonseca, M. Simoes, J. Leon, H. Roehl, M.L. Cancela, and A. Jacinto, Differentiated skeletal cells contribute to blastema formation during zebrafish fin regeneration. Development, 2011. 138(18): p. 3897-905

6. Ando, K., E. Shibata, S. Hans, M. Brand, and A. Kawakami, Osteoblast Production by Reserved Progenitor Cells in Zebrafish Bone Regeneration and Maintenance. Dev Cell, 2017. 43(5): p. 643650 e3

7. Yang, J., P. Antin, G. Berx, C. Blanpain, T. Brabletz, M. Bronner, K. Campbell, A. Cano, J. Casanova, G. Christofori, S. Dedhar, R. Derynck, H.L. Ford, J. Fuxe, A. Garcia de Herreros, G.J. Goodall, A.K. Hadjantonakis, R.J.Y. Huang, C. Kalcheim, R. Kalluri, Y. Kang, Y. Khew-Goodall, H. Levine, J. Liu, G.D. Longmore, S.A. Mani, J. Massague, R. Mayor, D. McClay, K.E. Mostov, D.F. Newgreen, M.A. Nieto, A. Puisieux, R. Runyan, P. Savagner, B. Stanger, M.P. Stemmler, Y. Takahashi, M. Takeichi, E. Theveneau, J.P. Thiery, E.W. Thompson, R.A. Weinberg, E.D. Williams, J. Xing, B.P. Zhou, G. Sheng, and E.M.T.I. Association, Guidelines and definitions for research on epithelial-mesenchymal transition. Nat Rev Mol Cell Biol, 2020. 21(6): p. 341-352. PMC7250738

8. Mani, S.A., W. Guo, M.J. Liao, E.N. Eaton, A. Ayyanan, A.Y. Zhou, M. Brooks, F. Reinhard, C.C. Zhang, M. Shipitsin, L.L. Campbell, K. Polyak, C. Brisken, J. Yang, and R.A. Weinberg, The epithelial-mesenchymal transition generates cells with properties of stem cells. Cell, 2008. 133(4): p. 704-15. PMC2728032

9. Saxena, K., M.K. Jolly, and K. Balamurugan, Hypoxia, partial EMT and collective migration: Emerging culprits in metastasis. Transl Oncol, 2020. 13(11): p. 100845. PMC7419667

10. Wong, A.Y. and J.L. Whited, Parallels between wound healing, epimorphic regeneration and solid tumors. Development, 2020. 147(1). PMC6983724

11. Gerber, T., P. Murawala, D. Knapp, W. Masselink, M. Schuez, S. Hermann, M. Gac-Santel, S. Nowoshilow, J. Kageyama, S. Khattak, J.D. Currie, J.G. Camp, E.M. Tanaka, and B. Treutlein, Single-cell analysis uncovers convergence of cell identities during axolotl limb regeneration. Science, 2018. 362(6413). PMC6669047

12. Leigh, N.D., G.S. Dunlap, K. Johnson, R. Mariano, R. Oshiro, A.Y. Wong, D.M. Bryant, B.M. Miller, A. Ratner, A. Chen, W.W. Ye, B.J. Haas, and J.L. Whited, Transcriptomic landscape of the blastema niche in regenerating adult axolotl limbs at single-cell resolution. Nat Commun, 2018. 9(1): p. 5153. PMC6279788

13. Ilvesaro, J., K. Metsikko, K. Vaananen, and J. Tuukkanen, Polarity of osteoblasts and osteoblastlike UMR-108 cells. J Bone Miner Res, 1999. 14(8): p. 1338-44

14. Okazaki, M., S. Takeshita, S. Kawai, R. Kikuno, A. Tsujimura, A. Kudo, and E. Amann, Molecular cloning and characterization of OB-cadherin, a new member of cadherin family expressed in osteoblasts. J Biol Chem, 1994. 269(16): p. 12092-8

15. Cheng, S.L., F. Lecanda, M.K. Davidson, P.M. Warlow, S.F. Zhang, L. Zhang, S. Suzuki, T. St John, and R. Civitelli, Human osteoblasts express a repertoire of cadherins, which are critical for BMP-2-induced osteogenic differentiation. J Bone Miner Res, 1998. 13(4): p. 633-44

16. Stains, J.P. and R. Civitelli, Cell-to-cell interactions in bone. Biochem Biophys Res Commun, 2005. 328(3): p. 721-7

17. Stewart, S., A.W. Gomez, B.E. Armstrong, A. Henner, and K. Stankunas, Sequential and opposing activities of Wnt and BMP coordinate zebrafish bone regeneration. Cell Rep, 2014. 6(3): p. 482-98. PMC4009375 
18. Hou, Y., H.J. Lee, Y. Chen, J. Ge, F.O.I. Osman, A.R. McAdow, M.H. Mokalled, S.L. Johnson, G. Zhao, and T. Wang, Cellular diversity of the regenerating caudal fin. Sci Adv, 2020. 6(33): p. eaba2084. PMC7423392

19. Cao, J., J.S. Packer, V. Ramani, D.A. Cusanovich, C. Huynh, R. Daza, X. Qiu, C. Lee, S.N. Furlan, F.J. Steemers, A. Adey, R.H. Waterston, C. Trapnell, and J. Shendure, Comprehensive single-cell transcriptional profiling of a multicellular organism. Science, 2017. 357(6352): p. 661667. PMC5894354

20. Alimperti, S. and S.T. Andreadis, $\mathrm{CDH} 2$ and $\mathrm{CDH11}$ act as regulators of stem cell fate decisions. Stem Cell Res, 2015. 14(3): p. 270-82. PMC4439315

21. Warzecha, C.C., T.K. Sato, B. Nabet, J.B. Hogenesch, and R.P. Carstens, ESRP1 and ESRP2 are epithelial cell-type-specific regulators of FGFR2 splicing. Mol Cell, 2009. 33(5): p. 591-601. PMC2702247

22. McMillan, S.C., J. Zhang, H.E. Phan, S. Jeradi, L. Probst, M. Hammerschmidt, and M.A. Akimenko, A regulatory pathway involving retinoic acid and calcineurin demarcates and maintains joint cells and osteoblasts in regenerating fin. Development, 2018. 145(11)

23. Galindo, M., J. Pratap, D.W. Young, H. Hovhannisyan, H.J. Im, J.Y. Choi, J.B. Lian, J.L. Stein, G.S. Stein, and A.J. van Wijnen, The bone-specific expression of Runx2 oscillates during the cell cycle to support a G1-related antiproliferative function in osteoblasts. J Biol Chem, 2005. 280(21): p. 20274-85. PMC2895256

24. San Martin, I.A., N. Varela, M. Gaete, K. Villegas, M. Osorio, J.C. Tapia, M. Antonelli, E.E. Mancilla, B.P. Pereira, S.S. Nathan, J.B. Lian, J.L. Stein, G.S. Stein, A.J. van Wijnen, and M. Galindo, Impaired cell cycle regulation of the osteoblast-related heterodimeric transcription factor Runx2-Cbfbeta in osteosarcoma cells. J Cell Physiol, 2009. 221(3): p. 560-71. PMC3066433

25. Farber, C.R., A. Reich, A.M. Barnes, P. Becerra, F. Rauch, W.A. Cabral, A. Bae, A. Quinlan, F.H. Glorieux, T.L. Clemens, and J.C. Marini, A novel IFITM5 mutation in severe atypical osteogenesis imperfecta type VI impairs osteoblast production of pigment epithelium-derived factor. J Bone Miner Res, 2014. 29(6): p. 1402-11. PMC4352343

26. Stephens, A.S., S.R. Stephens, C. Hobbs, D.W. Hutmacher, D. Bacic-Welsh, M.A. Woodruff, and N.A. Morrison, Myocyte enhancer factor 2c, an osteoblast transcription factor identified by dimethyl sulfoxide (DMSO)-enhanced mineralization. J Biol Chem, 2011. 286(34): p. 30071-86. PMC3191047

27. Qin, X., Q. Jiang, T. Miyazaki, and T. Komori, Runx2 regulates cranial suture closure by inducing hedgehog, Fgf, Wht and Pthlh signaling pathway gene expressions in suture mesenchymal cells. Hum Mol Genet, 2019. 28(6): p. 896-911

28. Aubin, I., C.P. Adams, S. Opsahl, D. Septier, C.E. Bishop, N. Auge, R. Salvayre, A. NegreSalvayre, M. Goldberg, J.L. Guenet, and C. Poirier, A deletion in the gene encoding sphingomyelin phosphodiesterase 3 (Smpd3) results in osteogenesis and dentinogenesis imperfecta in the mouse. Nat Genet, 2005. 37(8): p. 803-5

29. Street, K., D. Risso, R.B. Fletcher, D. Das, J. Ngai, N. Yosef, E. Purdom, and S. Dudoit, Slingshot: cell lineage and pseudotime inference for single-cell transcriptomics. BMC Genomics, 2018. 19(1): p. 477. PMC6007078

30. Govindan, J. and M.K. lovine, Dynamic remodeling of the extra cellular matrix during zebrafish fin regeneration. Gene Expr Patterns, 2015. 19(1-2): p. 21-9

31. Saelens, W., R. Cannoodt, H. Todorov, and Y. Saeys, A comparison of single-cell trajectory inference methods. Nat Biotechnol, 2019. 37(5): p. 547-554

32. Warzecha, C.C., P. Jiang, K. Amirikian, K.A. Dittmar, H. Lu, S. Shen, W. Guo, Y. Xing, and R.P. Carstens, An ESRP-regulated splicing programme is abrogated during the epithelialmesenchymal transition. EMBO J, 2010. 29(19): p. 3286-300. PMC2957203

33. Shapiro, I.M., A.W. Cheng, N.C. Flytzanis, M. Balsamo, J.S. Condeelis, M.H. Oktay, C.B. Burge, and F.B. Gertler, An EMT-driven alternative splicing program occurs in human breast cancer and modulates cellular phenotype. PLoS Genet, 2011. 7(8): p. e1002218. PMC3158048

34. Bebee, T.W., J.W. Park, K.I. Sheridan, C.C. Warzecha, B.W. Cieply, A.M. Rohacek, Y. Xing, and R.P. Carstens, The splicing regulators Esrp1 and Esrp2 direct an epithelial splicing program essential for mammalian development. Elife, 2015. 4. PMC4566030

35. Burguera, D., Y. Marquez, C. Racioppi, J. Permanyer, A. Torres-Mendez, R. Esposito, B. Albuixech-Crespo, L. Fanlo, Y. D'Agostino, A. Gohr, E. Navas-Perez, A. Riesgo, C. Cuomo, G. 
Benvenuto, L.A. Christiaen, E. Marti, S. D'Aniello, A. Spagnuolo, F. Ristoratore, M.I. Arnone, J. Garcia-Fernandez, and M. Irimia, Evolutionary recruitment of flexible Esrp-dependent splicing programs into diverse embryonic morphogenetic processes. Nat Commun, 2017. 8(1): p. 1799. PMC5703972

36. Tomita, K., A. van Bokhoven, G.J. van Leenders, E.T. Ruijter, C.F. Jansen, M.J. Bussemakers, and J.A. Schalken, Cadherin switching in human prostate cancer progression. Cancer Res, 2000. 60(13): p. 3650-4

37. Zeisberg, M. and E.G. Neilson, Biomarkers for epithelial-mesenchymal transitions. J Clin Invest, 2009. 119(6): p. 1429-37. PMC2689132

38. Hoffmann, I. and R. Balling, Cloning and expression analysis of a novel mesodermally expressed cadherin. Dev Biol, 1995. 169(1): p. 337-46

39. Kimura, Y., H. Matsunami, T. Inoue, K. Shimamura, N. Uchida, T. Ueno, T. Miyazaki, and M. Takeichi, Cadherin-11 expressed in association with mesenchymal morphogenesis in the head, somite, and limb bud of early mouse embryos. Dev Biol, 1995. 169(1): p. 347-58

40. Clendenon, S.G., B. Shah, C.A. Miller, G. Schmeisser, A. Walter, V.H. Gattone, 2nd, K.F. Barald, Q. Liu, and J.A. Marrs, Cadherin-11 controls otolith assembly: evidence for extracellular cadherin activity. Dev Dyn, 2009. 238(8): p. 1909-22. PMC2773293

41. Clendenon, S.G., S. Sarmah, B. Shah, Q. Liu, and J.A. Marrs, Zebrafish cadherin-11 participates in retinal differentiation and retinotectal axon projection during visual system development. Dev Dyn, 2012. 241(3): p. 442-54

42. Manohar, S., A. Camacho-Magallanes, C. Echeverria, Jr., and C.D. Rogers, Cadherin-11 Is Required for Neural Crest Specification and Survival. Front Physiol, 2020. 11: p. 563372. PMC7662130

43. Jolly, M.K., B.T. Preca, S.C. Tripathi, D. Jia, J.T. George, S.M. Hanash, T. Brabletz, M.P. Stemmler, J. Maurer, and $\mathrm{H}$. Levine, Interconnected feedback loops among ESRP1, HAS2, and CD44 regulate epithelial-mesenchymal plasticity in cancer. APL Bioeng, 2018. 2(3): p. 031908. PMC6324214

44. Tapial, J., K.C.H. Ha, T. Sterne-Weiler, A. Gohr, U. Braunschweig, A. Hermoso-Pulido, M. Quesnel-Vallieres, J. Permanyer, R. Sodaei, Y. Marquez, L. Cozzuto, X. Wang, M. GomezVelazquez, T. Rayon, M. Manzanares, J. Ponomarenko, B.J. Blencowe, and M. Irimia, An atlas of alternative splicing profiles and functional associations reveals new regulatory programs and genes that simultaneously express multiple major isoforms. Genome Res, 2017. 27(10): p. 17591768. PMC5630039

45. Lee, H.J., Y. Hou, Y. Chen, Z.Z. Dailey, A. Riddihough, H.S. Jang, T. Wang, and S.L. Johnson, Regenerating zebrafish fin epigenome is characterized by stable lineage-specific DNA methylation and dynamic chromatin accessibility. Genome Biol, 2020. 21(1): p. 52. PMC7047409

46. Liberzon, A., C. Birger, H. Thorvaldsdottir, M. Ghandi, J.P. Mesirov, and P. Tamayo, The Molecular Signatures Database (MSigDB) hallmark gene set collection. Cell Syst, 2015. 1(6): p. 417-425. PMC4707969

47. Roca, H., M. Pande, J.S. Huo, J. Hernandez, J.D. Cavalcoli, K.J. Pienta, and R.C. McEachin, $A$ bioinformatics approach reveals novel interactions of the OVOL transcription factors in the regulation of epithelial - mesenchymal cell reprogramming and cancer progression. BMC Syst Biol, 2014. 8: p. 29. PMC4008156

48. Pfefferli, C. and A. Jazwinska, The art of fin regeneration in zebrafish. Regeneration (Oxf), 2015. 2(2): p. 72-83. PMC4895310

49. Stemmler, M.P., R.L. Eccles, S. Brabletz, and T. Brabletz, Non-redundant functions of EMT transcription factors. Nat Cell Biol, 2019. 21(1): p. 102-112

50. Li, R., J. Liang, S. Ni, T. Zhou, X. Qing, H. Li, W. He, J. Chen, F. Li, Q. Zhuang, B. Qin, J. Xu, W. Li, J. Yang, Y. Gan, D. Qin, S. Feng, H. Song, D. Yang, B. Zhang, L. Zeng, L. Lai, M.A. Esteban, and D. Pei, A mesenchymal-to-epithelial transition initiates and is required for the nuclear reprogramming of mouse fibroblasts. Cell Stem Cell, 2010. 7(1): p. 51-63

51. Cieply, B., J.W. Park, A. Nakauka-Ddamba, T.W. Bebee, Y. Guo, X. Shang, C.J. Lengner, Y. Xing, and R.P. Carstens, Multiphasic and Dynamic Changes in Alternative Splicing during Induction of Pluripotency Are Coordinated by Numerous RNA-Binding Proteins. Cell Rep, 2016. 15(2): p. 247-55. PMC5718363 
52. Liu, X., H. Sun, J. Qi, L. Wang, S. He, J. Liu, C. Feng, C. Chen, W. Li, Y. Guo, D. Qin, G. Pan, J. Chen, D. Pei, and H. Zheng, Sequential introduction of reprogramming factors reveals a timesensitive requirement for individual factors and a sequential EMT-MET mechanism for optimal reprogramming. Nat Cell Biol, 2013. 15(7): p. 829-38

53. Wang, H. and J.J. Unternaehrer, Epithelial-mesenchymal Transition and Cancer Stem Cells: At the Crossroads of Differentiation and Dedifferentiation. Dev Dyn, 2019. 248(1): p. 10-20

54. Battula, V.L., K.W. Evans, B.G. Hollier, Y. Shi, F.C. Marini, A. Ayyanan, R.Y. Wang, C. Brisken, R. Guerra, M. Andreeff, and S.A. Mani, Epithelial-mesenchymal transition-derived cells exhibit multilineage differentiation potential similar to mesenchymal stem cells. Stem Cells, 2010. 28(8): p. 1435-45. PMC3523728

55. Butler, A., P. Hoffman, P. Smibert, E. Papalexi, and R. Satija, Integrating single-cell transcriptomic data across different conditions, technologies, and species. Nat Biotechnol, 2018. 36(5): p. 411-420. PMC6700744

56. Bunis, D.G., J. Andrews, G.K. Fragiadakis, T.D. Burt, and M. Sirota, dittoSeq: Universal UserFriendly Single-Cell and Bulk RNA Sequencing Visualization Toolkit. Bioinformatics, 2020. PMC8016464 